\title{
Air Distributor Designs for Fluidized Bed Combustors: A Review
}

\author{
Ahmmad Shukrie \\ Energy Sustainability Focus Group \\ Faculty of Mechanical Engineering \\ Universiti Malaysia Pahang \\ Malaysia \\ shukrie@ump.edu.my
}

\author{
Shahrani Anuar \\ Energy Sustainability Focus Group \\ Faculty of Mechanical Engineering \\ Universiti Malaysia Pahang \\ Malaysia \\ shahrani@ump.edu.my
}

\author{
Ahmed Nurye Oumer \\ Energy Sustainability Focus Group \\ Faculty of Mechanical Engineering \\ Universiti Malaysia Pahang \\ Malaysia \\ nurye@ump.edu.my
}

\begin{abstract}
Fluidized bed combustion (FBC) has been recognized as one of the suitable technologies for converting a wide variety of biomass fuels into energy. One of the key factors affecting the successful operation of fluidized bed combustion is its distributor plate design. Therefore, the main purpose of this article is to provide a critical overview of the published studies that are relevant to the characteristics of different fluidized bed air distributor designs. The review of available works display that the type of distributor design significantly affects the operation of the fluidized bed i.e., performance characteristics, fluidization quality, air flow dynamics, solid pattern and mixing caused by the direction of air flow through the distributors. Overall it is observed that high pressure drop across the distributor is one of the major draw backs of the current distributor designs. However, fluidization was stable in a fluidized bed operated at a low perforation ratio distributor due to the pressure drop across the distributor, adequate to provide uniform gas distribution. The swirling motion produced by the inclined injection of gas promotes lateral dispersion and significantly improves fluidization quality. Lastly, the research gaps are highlighted for future improvement consideration on the development of efficient distributor designs.
\end{abstract}

Keywords-Fluidized bed combustor; air distributor design; swirling distributor; perforated distributor

\section{INTRODUCTION}

Fluidized bed combustion has been recognized as a suitable technology for converting a wide variety of fuels into energy. One of the key features offered by fluidized bed combustion is the reduced emission of $\mathrm{SO}_{2}$ and $\mathrm{NO}_{\mathrm{x}}$. The noxious combustion products released from the combustion of hydrocarbon fuels are captured by the calcined limestone for easy handling and disposal. Unlike many others energy conversion technologies such as gasification and pyrolysis, the combustion efficiency in a fluidized bed can reach up to $99 \%$ resulted from constant temperature distribution and adequate amount of oxygen supplied [1].

Depending on the distributors design in a fluidized bed, different types of particles with different sizes and shapes can be used. For instance, the fluidized bed combustor (Figure 1), which consists of the following: combustion chamber, distributor plate, bed pre-heater, gas outlet, and fuel feeding system, uses inert particles, usually alumina and river sands, as its bed materials. The inert materials are preheated to the operating temperature by the pre-heater, and this causes the particles bed to absorb and store high amounts of heat. Fluidizing air is passed through the distributor plate, and when the air velocity reaches a particular point on which the gravity, the weight of the particle and the overall weight of the bed are balanced, the bed is suspended and exhibits a fluid-like property. Then, the bed is said to be fluidized and the velocity at this particular point is the minimum fluidization velocity.

One of the key parameters that affect the successful operation of fluidized beds is the type of the distributor plate. It is known that the efficient and stable operation of a fluidized bed is sensitively controlled by the design of the distributor [2, 3]. The hydrodynamics of flow in the dense phase, quality of gas dispersion, bubble size and its behaviour, gas-solids contacting, gas hold-up, residence time distribution of gas, solid movement and mixing pattern are some of the aspects that are affected by the distributor design $[4,5]$.

The objective of this paper is to give an overview on past and present works related to the characteristics of different distributor designs in fluidized beds.

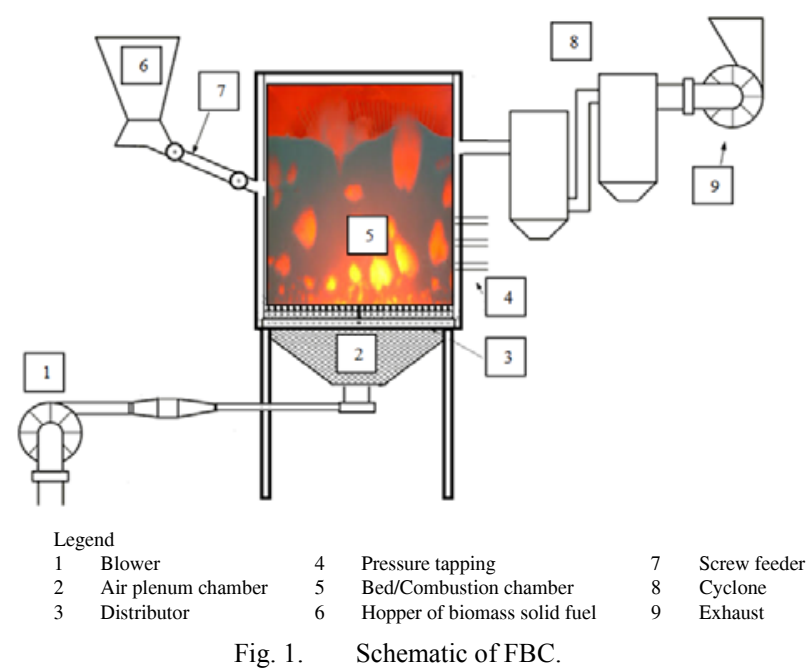




\section{DISTRIBUTOR DESIGNS}

Figure 2 summarizes the various types of distributor designs available in the literature. The distributors are grouped based on the direction of air entering into the distributor (normal direction, lateral direction and inclined direction). Each distributor's functionality and its applications are discussed in detail in the following sections.

\section{A. Normal Direction of Air Entrance}

A wide range of distributor plates available are designed in such a way that they inject the air into the fluidized bed in a normal direction to the distributor plate. The main types are: (i) perforated plate (ii) sparger and (iii) sintered metal distributors.

\section{(i) Perforated Plate Distributor}

Perforated plate distributor consists of holes arranged in either square or triangular pitches that are distributed over the distributor's area. The percentage opening area, or some might refer it as perforated ratio, is calculated by dividing the total number of holes area over the distributor area. The effect of distributor plates on the distribution of temperature profiles along the axis of the combustion chamber during the combustion of low density rice husk fuels in a fluidized bed was studied in [6]. Two different distributors with perforated ratios of $2.6 \%$ and $7.6 \%$ were tested. The peak temperature points - as an indication for combustion of fuel particles were found at $25 \mathrm{~cm}$ and $60 \mathrm{~cm}$ above the distributors for $2.6 \%$ and $7.6 \%$ perforated ratio distributors respectively. The low bulk density of the fuel (rice husks) that prone to float to a certain height resulted to the increment of peak temperature profile in the freeboard area. However, no specific discussion pertaining on the effect of perforated ratio on temperature variation was highlighted by the authors.

In [7] the importance of perforated ratios on the distributor and bed pressure drop, solid holdup, and gas-solid behavior was investigated. Three different types of perforated distributors having $0.46 \%, 0.86 \%$ and $1.10 \%$ perforated ratios were investigated by utilizing the CFD simulation and comparison with experimental and available published data.
The results showed that as superficial velocity progressed, the distributor pressure drop increased with decreasing perforated ratio due to increase of airflow resistance. However, solid circulation and gas-solid distribution was recorded more homogenous in the bed of $0.46 \%$ perforated ratio than that of $0.86 \%$ and $1.10 \%$. The authors concluded that fluidization was more stable in the bed operated in the low perforated ratio distributor due to the pressure drop across the distributor being adequate to provide uniform gas distribution.

\section{(ii) Sparger}

Various sparger designs are available such as radial pipes and multiple rings [8-11]. Air is uniformly distributed to all the holes through the main air inlet or header. Comparison between different sparger configurations has been conducted by many researchers to find the optimum sparger type and design. For instance, systematic procedures for the selection of sparger design and type were highlighted in [8]. In that study, a new wheel-type sparger was proposed based on the finding that conventional type spargers provide highly non-uniform flow. More recently, the effect of entrance configurations on the flow field in an industrial fluidized bed reactor were the primary interest of some researchers [5, 12]. The Computational Fluid Dynamics-Population Balance Model (CFD-PBM) coupled model was implemented to investigate the effect of a sparger distributor on reactor performance. Comparison of reactor performances in terms of bubble formation and dead zone formation were made with a perforated distributor. The image processing analysis of the bubble phase show that the bubble size distribution in the case of the sparger distributor was more uniform than that of the perforated plate. The CFD-PBM simulation results however showed that dead zones were spotted in the corners of the sparger distributor due to particles clustering. The authors concluded that the perforated distributor was found to be more advantageous than the sparger-type distributor. The jet formation above the perforated distributor due to the restricted air passages provides adequate driving force and thus eliminates the cluster formation, resulting in more heterogeneous flow field and better gas-solid interaction.

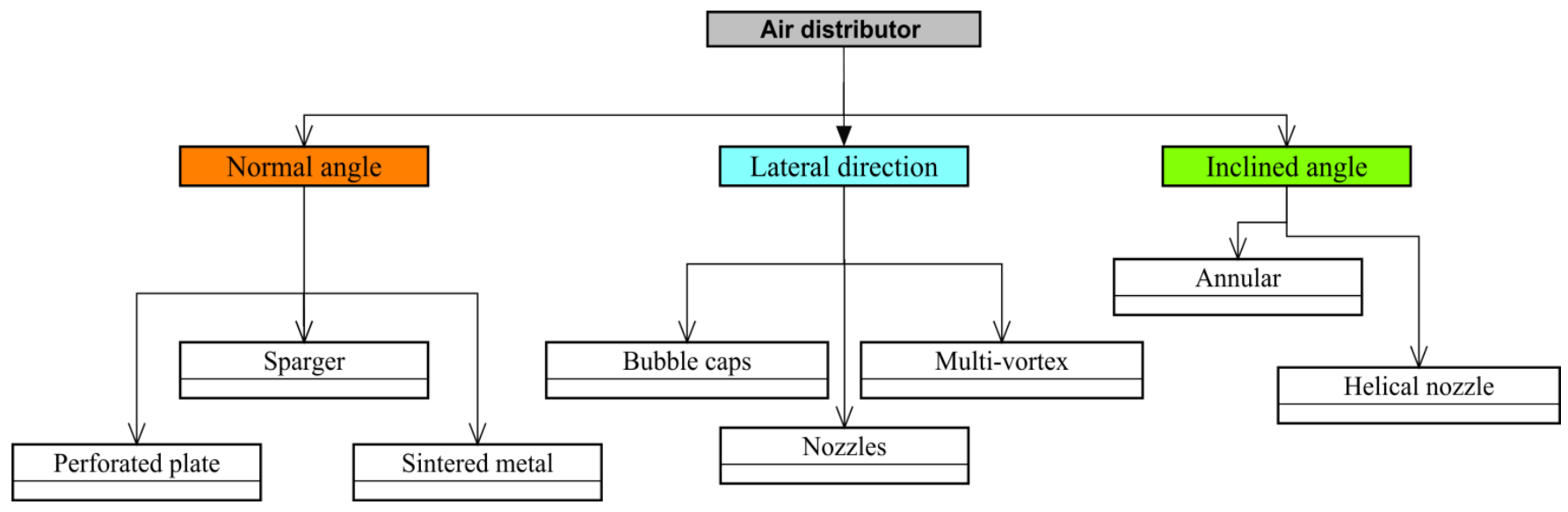

Fig. 2. Summary of primary distributor designs available in the literature. 


\section{(iii) Sintered Metal}

Sintered metal distributor (SMD) is made from multiple layers of woven wire mesh of comparatively small aperture and high open area ratio compared to perforated-type distributor. The effect of SMD with different apertures and open area ratios on the fluidization quality of air dense medium fluidized bed separator was investigated in [2]. Five SMDs of aperture sizes and open area ratios ranging from 5-10 $\mu \mathrm{m}$ and $25-40 \%$ were employed in the experiments. Results showed that the quality and stability of fluidization over a wide range of air velocity was achieved by using a smaller aperture size of $5 \mu \mathrm{m}$ SMD with a bigger open area ratio of $40 \%$ due to the increase of pressure drop. The effect of different SMD aperture sizes on the solid flow structure in a bubbling fluidized bed from [13] showed that there exist four solid flow patterns in a bubbling fluidized bed, influenced by the distributor aperture size and the bed aspect ratio (H/D). The solid flow patterns and behaviors were observed by using the positron emission particle tracking (PEPT) technique. Five different aperture sizes: $1 \mu \mathrm{m}, 10 \mu \mathrm{m}, 15 \mu \mathrm{m}, 60 \mu \mathrm{m}$ and $230 \mu \mathrm{m}$ of open area ratios ranging from $24 \%$ to $30 \%$ were investigated with $1,1.5$ and 2 bed aspect ratios resulted in more than 25 sets of experiments. Poor solid circulation patterns was observed with single unit bed aspect ratio fluidized bed operated with SMD of 15, 60 and $230 \mu \mathrm{m}$ aperture sizes. From the results, it was concluded that the desirable flow patterns in a fluidized bed that were very much related to particles' kinetic energy were the combination of superficial air velocity, bed aspect ratio and distributor aperture size.

\section{B. Lateral Direction of Air Entrance}

Some types of distributor plates are designed in such a way that the fluidizing air is injected into the fluidized beds in lateral direction. The main types are: (i) bubble-cap, (ii) nozzles and (ii) multi-vortex type distributors.

\section{(i) Bubble-cap}

Bubble cap type distributor consists of orifices attached with caps in the upper part of the orifice plate to avoid the accumulation of stationary solids and particles fall back. The fluidizing air is flowing through the holes in a lateral direction beneath the caps in a way that the solid fall through the holes is prevented by high velocity jets. The distributor free open area is low and results in high pressure drop through the distributor. The distributor effect at the bottom region of the fluidized bed during turbulent fluidization was studied in [14]. Experimental results by using a bubble cap distributor showed that the axial flow structure at the bottom region of the bubbling fluidized bed was more stable. Comparison was made with the perforated plate distributor that showed opposite pressure variation, though both having the same open area ratio and similar distributor pressure drop profile. The possible reason behind this was the jetting effect through the perforated holes and the formation of dead zones through the perforated interstices. The entrainment rate however was found to be higher in the perforated plate distributor than that of the bubble cap distributor given the same superficial velocity when operated at the transition region from bubbling to turbulent fluidization. This indicates that the bubble formation from lateral jets through the bubble cap reduce the entrainment rate compared to the perforated plate.

Motivated by the fact that none of the literature available pertaining on the performance of the distributors at higher operating temperature, Sánchez-Prieto et al [15] compared the performance of two types of distributors, bubble cap and perforated distributors up to $300^{\circ} \mathrm{C}$ operating temperature. Considering that both distributors have a similar free open area of $0.8 \%$, distributor pressure drop was found to be higher in the bubble cap distributor measured at ambient and higher operating temperatures due to a lower discharge coefficient compared to that of the perforated plate distributor. At the same air velocity, pressure drops for both distributors were recorded to decrease as the operating temperature increases due to the decrement of gas density. Moreover, a new methodology was developed in designing the distributor plate for higher operating temperatures. The parameters that have shown to have significant effect on the distributor performance at higher operating temperatures were the distributor free open area ratio, the bed aspect ratio and the pressure drop ratio. However, no distinctive comparison regarding the effect of distributors on bed behavior particularly on particulate mixing operated at higher operating temperatures was made by the authors.

\section{(ii) Nozzles}

The nozzles were either mounted on the distributor plate with inverted ' $L$ ' shapes or with some inclination angles. The significant difference between nozzles and bubble cap type distributors is that no caps are installed on top of the nozzles and the solely prevention method of particle fall back into the plenum is by the lateral jet flow from the nozzles. Horizontal gas dispersion in a bubbling fluidized bed was investigated in [16] by using response surface methodology (RSM). Three nozzle distributors with different open area ratios of $1 \%, 2.5 \%$ and $4 \%$ were employed in the experiments. Three different sizes of glass beads of $360 \mu \mathrm{m}, 460 \mu \mathrm{m}$ and $545 \mu \mathrm{m}$ were used as bed materials in a rectangular shaped bubbling fluidized bed. The horizontal gas dispersion was further compared with two other types of distributors; bubble cap and perforated fabricated with the same open area ratios as the nozzle distributor. The coefficient of horizontal dispersion was assigned as an objective function for the three types of distributors and was calculated from tracer concentration. The higher value of coefficient of horizontal dispersion implies better horizontal gas dispersion and thus better mixing. The perforated distributor was recorded to have a low value of dispersion coefficient for all operations. No distinctive difference observed in the dispersion coefficient between the nozzle and the bubble cap distributors at low operating velocity. At higher operating velocities the coefficient of horizontal dispersion for the nozzle type was higher compared to the bubble cap and perforated types. This suggests that the dispersion in the bubbling fluidized bed can be enhanced by increasing the operating velocity. Authors concluded that the distributor design was the dominant factor for gas dispersion in the fluidized bed. 
Computational Fluid Dynamics (CFD) simulation on the hydrodynamics of the bubbling fluidized bed gasifier with a nozzle-type gas distributor was studied in [17]. Two cases of air distribution (one ideal and one realistic) with the nozzletype distributor were considered in their 3D CFD simulations. In the ideal case, uniform gas injection was located at the bottom of the fluidized bed (without considering the individual nozzle distributors). The realistic case, on the other hand, dealt with full scale nozzle geometry. The geometry was further simplified for CFD analysis. Significant differences were recorded for the two cases in terms of solid volume fraction (SVF), uniformity index (UI) and solid stack volume (SSV). Gas flow streamline from CFD simulation showed that the gas flow was more uniform in the ideal case. SVF was measured higher in the realistic case due to the non-uniform flow distribution where some dense and stationary solid zones were observed at the bottom zone of the fluidized bed, corners and between nozzle distributors. The UI along the fluidized bed height for the realistic case was lower about 5\% than that of the ideal case. The UI of unity represents even solid distribution in a specified surface area. The SSV that measures the dead zones region was found lower in the ideal case. It was shown that the SSV has to be identified and minimized when modeling fluidized-beds with real distributors because it may lead to serious operating problems such as slugging and channeling due to blocking of distributors. Modeling the fluidized bed with ideal air entering the system showed significant deviation from the real phenomenon. Thus, the actual distributor geometry should be considered in modeling the air distribution in fluidized bed system.

\section{(ii) Multi-vortex type distributors}

A novel multi-vortex distributor was introduced in [18] and it consisted of 38 unit of 1/6 inch outside diameter (OD) tubes with different orientations arranged in a rectangular pitch. According to the authors, the proposed tubes would theoretically improve the horizontal momentum and eliminate the problem associated with dead zones at the surface of the distributor and at the bottom region of the bed. This would in turn intensify the lateral dispersion and improve mass transfer. The gas dispersion was compared to the conventional perforated plate distributor that consisted of 35 perforated holes of $2 \mathrm{~mm}$ internal diameter (ID). The conversion efficiency, defined as experimentally measured conversion over the ideal maximum conversion, was used as a comparison parameter. Conversion efficiency of unity attributed to better interfacial and axial dispersion. For all inlet velocities, it was found that the multi-vortex distributor improved the conversion effeciencies by $14.75 \%$ compared to the perforated plate distributor. An unexpected increase in bubble size however was observed in multi vortex distributor in a factor of 1.4 and 1.5 from visual and pressure fluctuation measurements. The authors argued that the increment of bubble size indicates an increment of mass transfer mechanism which was later associated with an increment of conventional and diffusional components by using multi-vortex distributor. However, no quantification was made by the authors to support the validity of the arguments.

\section{Inclined Direction of Air Entrance}

The inclined direction of air entering into the fluidized bed at an angle $\theta$ produces swirling air motion as a result of velocity $U$ entering the bed which consists of two velocity components: U $\cos \theta$ which is responsible for fluidization and Usin $\theta$ that induced swirling air motion inside the fluidized bed. The main types of inclined distributors are: (i) annular inclined blades, and (ii) helical nozzle distributors.

\section{(i) Annular inclined blades}

The annular distributor consists of blades arranged at $\alpha$ certain inclination angle. The overlapping length between the blades directs the air at the desired angle. The centrifugal force is a result of a swirling motion of bed material in a circular path which is imparted by the tangential component of air velocity due to the inclined angle of the distributor blades. The original idea of annular inclined blades for fluidized bed can be traced back to [19]. The annular inclined distributor consists of $\mathrm{N}$ number of overlapping sector-shaped blades. The performance of the distributor was evaluated as a function of number of blades and fraction free area. Comparison was made with the sintered metal distributor by using a heat transfer coefficient between immersed heater and bed materials as an objective parameter. Significant improvement of the heat transfer coefficient of the bed materials was achieved by using inclined blades compared to that of the sintered metal distributor at higher operating velocity. Changing fraction free area from $1.2 \%$ to $4.1 \%$ seemed to have no effect on the heat transfer coefficient but changing the number of blades from 24 to 32 units showed a significant effect. Moreover, the correlation that governs the heat transfer coefficient and the distributor geometry was developed.

Following the annular inclined distributor of Ouyang and Levenspiel, the work of some researchers was devoted to understand the hydrodynamics of swirling fluidized beds [2029]. The annular inclined blades of Ouyang and Levenspiel was modified into truncated sectors of a circle that resulted in arrangement of blades of annular inclined distributor [22]. Varying the distributor angle (sector) from $12^{\circ}$ to $15^{\circ}$ and $18^{\circ}$ was shown to have a significant effect on particle velocity [23]. Generally, the particles velocity was reduced by $18 \%$ for every $3^{\circ}$ angle increment. Increment of the distributor angle makes the distributor sector to become wider thus results in higher surface friction due to larger surface area of particles in contact with the distributor sector. The inclined arrangement of blades was shown to move the air radially towards the peripheral of the bed. Eventually an insignificant dead-region was created at the center region of fluidized bed that required the installation of a cone. In [20] it was reported that the installation of a central cone reduced the superficial air velocity from the distributor to the free surface of the bed due to the increasing cross section available for air flow.

Application of annular inclined distributors in the fluidized bed combustion are documented in the works of many researchers $[25,26,30]$. An innovative swirling fluidized bed combustor (SFBC) was used to burn $80 \mathrm{~kg} / \mathrm{hr}$ rice husk of different moisture contents of $8.4 \%$ to $35 \%$ and $20 \%-80 \%$ of excess air [26]. The primary air for combustion was supplied through an annular inclined distributor that induced swirling 
flow motion in a combustion chamber. Constant axial temperature profiles were recorded along the height of SFBC that reflects effectiveness of the combustion by using annular inclined distributor; in contrast to the inconsistent axial temperature profile recorded during the combustion of rice husk in [6] using a perforated plate distributor. It is thus anticipated that the swirling distributor holds promise for industrial fluidized bed applications.

\section{(ii) Helical nozzle distributors}

A novel spiral helix nozzle distributor introduced in [31] was shown to induce swirling air motion as the air enters the fluidized bed. The swirling distributor consists of a $5 \mathrm{~mm}$ diameter rod with six-turn helical coil attached in a $10 \mathrm{~mm}$ diameter nozzle. Besides producing a swirling air motion, the functionality of the helix was to retain the particles when the fluidized bed is at a de-fluidized state. The spiral helix nozzle was attached together with 61 perforated holes of $0.4 \mathrm{~mm}$ diameter on a $50 \mathrm{~mm}$ diameter distributor plate. Different case studies were conducted on which the distributors were modified into three configurations: plain nozzle, lowered helix and flush helix. The formation of jets and bubbles from these configurations were observed and recorded by using ultra-fast magnetic resonance imaging (MRI). The experimental results indicated that the swirling motion produced by novel helicaltype gas distributors promotes lateral dispersion and significantly improves the fluidization quality compared to a plain nozzle without a spiral. However, the presence of the helical coils in the distributor significantly increases the pressure drop across the distributor.

\section{FINDINGS AND RESEARCH GAPS} below:

The critical findings from the literature review are listed

1. The major factors which might affect the intensity of mixing include fluidizing velocity, particle size and arrangement of the gas flow through the distributor.

2. The parameters that have shown to have a significant effect on the distributor's performance at higher operating temperatures were the distributor free open area ratio, the bed aspect ratio and the pressure drop ratio.

3. High pressure drop across the distributor is one of the major draw backs of the current distributor designs.

4. Fluidization was more stable in the bed operated in a low perforated ratio distributor due to adequate pressure drop across the distributor to provide uniform gas distribution.

5. The swirling motion produced by inclined injection of gas promotes lateral dispersion and significantly improves the fluidization quality.

It is thus anticipated that the performance of the current fluidized beds can be further improved through the development of new distributor designs. Moreover, the swirling motion produced by the inclined injection of air was shown to improve particle mixing. However, the current swirling distributor designs used overlapping blades that limits the application of fluidization only to larger bed size materials. Besides, the recent attempt on inducing swirling flow by using helical coil was proven to increase the distributor pressure drop; which is a drawback in the distributor design. The fluidization was found more stable in the bed operated by a perforated distributor due to the adequate pressure drop across the distributor to provide uniform gas distribution. Therefore, a new distributor design is in need by integrating the perforated and swirling distributors. Considering most of the fluidization processes are operated with pressurized air as a working fluid, attempts have to be made in future studies to investigate the effect of different distributor designs on the hydrodynamics of the fluidized bed operated by a low pressure blower.

\section{CONCLUSION}

Overview on past and present works related to the characteristics of different distributor designs in fluidized bed were critically reviewed and discussed in this paper. The parameters that affect the performance of fluidized bed were identified and research gaps pertaining to the distributor designs were highlighted for improvement consideration on future developments of distributor designs for fluidized bed combustors. This review indicated that the efficient operation of a fluidized bed is dependent on distributor performance, which, in turn, is dependent on the design parameters of the distributor. In general, the incorporation of the right distributor design in a fluidization operation would represent inexpensive means to offer better economic and technical performance for future developments of fluidized bed combustors.

\section{ACKNOWLEDGMENT}

The authors wish to express their gratitude to the Faculty of Mechanical Engineering and Research and Innovation Department, Universiti Malaysia Pahang for providing the research facilities and supporting the research under University Research Grant RDU140355 and Post Graduate Research Scheme (PGRS) GRS140346.

\section{REFERENCES}

[1] J. Han, H. Kim, W. Minami, T. Shimizu, G. Wang, "The effect of the particle size of alumina sand on the combustion and emission behavior of cedar pellets in a fluidized bed combustor", Bioresour. Technol., Vol. 99, pp. 3782-37866, 2008

[2] X. Yang, Y. Zhao, Z. Luo, Z. Chen, S. Song, "Effects of sintered metal distributor on fluidization quality of the air dense medium fluidized bed", Min. Sci. Technol., Vol. 21, pp. 681-685, 2011

[3] K. Vakhshouri, J. R. Grace, "Effects of the plenum chamber volume and distributor geometry on fluidized bed hydrodynamics", Particuology, Vol. 8, pp. 2-12, 2010

[4] L. Huilin, Z. Yunhua, J. Ding, Z. Linyan, L. Yaning, S. Ana, "Numerical Modeling of Gas Tubular Distributors in Bubbling Fluidized-Bed Incinerators", Ind. Eng. Chem. Res., Vol. 45,pp. 6818-6827, 2006

[5] V. Akbari, T. N. G. Borhani, R. Aramesh, M. K. A. Hamid, A. Shamiri, M. A. Hussain, "Evaluation of hydrodynamic behavior of the perforated gas distributor of industrial gas phase polymerization reactor using CFDPBM coupled model”, Comput. Chem. Eng., Vol. 82, pp. 344-361, 2015

[6] K. V. N. S. Rao, G. V. Reddy, "Effect of Distributor Design on Temperature Profiles in Fluidized Bed During the Combustion of Rice Husk", Combust. Sci. Technol., Vol. 179, pp. 1589-1603, 2007

[7] S. Dong, C. Cao, C. Si, Q. Guo, "Effect of Perforated Ratios of Distributor on the Fluidization Characteristics in a Gas-Solid Fluidized Bed”, Ind. Eng. Chem. Res., Vol. 48, pp. 517-527, 2009

[8] A. V. Kulkarni, J. B. Joshi, "Design and selection of sparger for bubble column reactor. Part I: Performance of different spargers", Chem. Eng. Res. Des., Vol. 89, pp. 1972-1985, 2011 
[9] A. V. Kulkarni, J. B. Joshi, "Design and selection of sparger for bubble column reactor. Part II: Optimum sparger type and design", Chem. Eng. Res. Des., Vol. 89, pp. 1986-1995, 2011

[10] B. N. Thorat, A. V. Shevade, K. N. Bhilegaonkar, R. H. Aglawe, U. Parasu Veera, S. S. Thakre, A. B. Pandit, S .B. Sawant, J. B. Joshi "Effect of Sparger Design and Height to Diameter Ratio on Fractional Gas Hold-up in Bubble Columns", Chem. Eng. Res. Des., Vol. 76, pp. 823-834, 1998

[11] M. T. Dhotre, K. Ekambara, J. B. Joshi, "CFD simulation of sparger design and height to diameter ratio on gas hold-up profiles in bubble column reactors", Exp. Therm. Fluid Sci., Vol. 28, pp. 407-421, 2004

[12] V. Akbari, T. N. G. Borhani, H. R. Godini, M. K. A. Hamid, "Modelbased analysis of the impact of the distributor on the hydrodynamic performance of industrial polydisperse gas phase fluidized bed polymerization reactors", Powder Technol., Vol. 267, pp. 398-411, 2014

[13] Y. Li, H. Fan, X. Fan, "Identify of flow patterns in bubbling fluidization", Chem. Eng. Sci., Vol. 117, pp. 455-464, 2014

[14] C. Sobrino, N. Ellis, M. de Vega, "Distributor effects near the bottom region of turbulent fluidized beds", Powder Technol., Vol. 189, pp. 2533, 2009

[15] J. Sánchez-Prieto, A. Soria-Verdugo, J. V. Briongos, D. Santana, "The effect of temperature on the distributor design in bubbling fluidized beds", Powder Technol., Vol. 261, pp. 176-184, 2014

[16] C. -S. Chyang, Y. -L. Han, C. -H. Chien, "Gas dispersion in a rectangular bubbling fluidized bed", J. Taiwan Inst. Chem. Eng., Vol. 41, pp. 195-202, 2010

[17] S. I. Ngo, Y. -I. Lim, B. -H. Song, U. -D. Lee, J. -W. Lee, J. -H. Song, "Effects of fluidization velocity on solid stack volume in a bubbling fluidized-bed with nozzle-type distributor", Powder Technol., Vol. 275, pp. 188-198, 2015

[18] H. G. Brink, J. Saayman, W. Nicol, "Two dimensional fluidised bed reactor: Performance of a novel multi-vortex distributor", Chem. Eng. J., Vol. 175, pp. 484-493, 2011

[19] F. Ouyang, O. Levenspiel, "Spiral Distributor for Fluidized Beds", Ind. Eng. Chem. Process Des. Dev., Vol. 25, pp. 504-507, 1986

[20] B. Sreenivasan, V. R. Raghavan, "Hydrodynamics of a swirling fluidised bed", Chem. Eng. Process. Process Intensif. Vol.41, pp. 99-106, 2002

[21] M. F. Mohideen, B. Sreenivasan, S. A. Sulaiman, V. R. Raghavan, "Heat transfer in a swirling fluidized bed with geldart type-D particles", Korean J. Chem. Eng., Vol. 29, pp. 862-867, 2012

[22] A. Shukrie, Studies on the residence time distribution of solids in a swirling fluidized bed, Masters thesis, Universiti Teknologi Petronas, 2012

[23] C. S. Miin, S. A. Sulaiman, V. R. Raghavan, M. R. Heikal, M. Y. Naz, "Hydrodynamics of multi-sized particles in stable regime of a swirling bed", Korean J. Chem. Eng., Vol. 32, pp. 2361-2367, 2015

[24] M. Faizal, S. M. Seri, M. Al-Hafiz, V. R. Raghavan, "CFD studies on velocity distribution of air in a swirling fluidized bed", Adv. Mater. Res., Vol. 468-471, pp. 25-29, 2012

[25] R. Kaewklum, V. I. Kuprianov, P. L. Douglas, "Hydrodynamics of airsand flow in a conical swirling fluidized bed: A comparative study between tangential and axial air entries", Energy Convers. Manag., Vol. 50, pp. 2999-3006, 2009

[26] V. I. Kuprianov, R. Kaewklum, K. Sirisomboon, P. Arromdee, S. Chakritthakul, "Combustion and emission characteristics of a swirling fluidized-bed combustor burning moisturized rice husk", Appl. Energy, Vol. 87, pp. 2899-2906, 2010

[27] P. Arromdee, V. I. Kuprianov, "A comparative study on combustion of sunflower shells in bubbling and swirling fluidized-bed combustors with a cone-shaped bed", Chem. Eng. Process. Process Intensif., Vol. 62, pp. 26-38, 2012

[28] A. S. M. Yudin, V. R. Raghavan, M. Narahari, "A mathematical model for residence time distribution analysis in swirling fluidized bed", Natl. Postgrad. Conf. (NPC), pp. 1-5, 2011

[29] R. Kaewklum, V. I. Kuprianov, "Experimental studies on a novel swirling fluidized-bed combustor using an annular spiral air distributor", Fuel, Vol. 89, pp. 43-52, 2010
[30] V. I. Kuprianov, R. Kaewklum, S. Chakritthakul, "Effects of operating conditions and fuel properties on emission performance and combustion efficiency of a swirling fluidized-bed combustor fired with a biomass fuel”, Energy, Vol. 36, pp. 2038-2048, 2011

[31] S. M. Aworinde, D. J. Holland, J. F. Davidson, "Investigation of a swirling flow nozzle for a fluidised bed gas distributor", Chem. Eng. Sci., Vol. 132, pp. 22-31, 2015 